\title{
Les arts peuvent-ils et doivent-ils « unir leurs forces »?
}

\author{
François-Charles Gaudard
}

Université de Toulouse Jean-Jaurès / IRPALL

\section{Abstract}

Der Symbolismus wirkte als Bahnbrecher in den Kunstbewegungen des 20. Jahrhunderts. Auf die Belle Époque folgten der Bruch, den der Erste Weltkrieg mit sich brachte, und Versuche, die Welt neu zu gestalten (wissenschaftliche Forschungen, mannigfaltige Kunstexperimente, Dialog zwischen den Künsten). Während es einerseits Versuche gegeben hat, die Wunden des Krieges mit Hilfe von Zeugenschaften und Gedenkfeiern zu heilen, zogen es manche Künstler vor, sich radikal von der Vergangenheit zu distanzieren (Dadaisten) und eine neue Wirklichkeit hervorzurufen (Surrealisten). Der Akzent wird hier auf die rege Tätigkeit in Paris (Poesie, Malerei, Musik, Tanz) gelegt sowie auf das revolutionäre russische Ballett, um die Wiederbelebung und Erneuerung im Denken und Schaffen zu dokumentieren. Der innovative, ja provozierende Dialog der Künste im Krieg und in der unmittelbaren Nachkriegszeit trug dazu bei, anders zu trauern und eine alternative Zukunft glaubwürdig zu machen.

Le fil conducteur de l'article est « le rôle fondateur du symbolisme " dans les mouvements artistiques du XXe siècle. À la Belle Époque succèdent la fracture de la guerre et les tentatives de recomposition du monde (exploration du monde et de l'homme dans les sciences, foisonnement des mouvements artistiques, pratique du dialogue entre les arts). Si une première tentative de « cicatrisation » se manifesta dans les témoignages et les commémorations, une autre solution consista à faire table rase du passé (dadaïsme) et à s'orienter vers la « surréalité ». L'accent est ici mis sur l'effervescence du milieu parisien (poésie, peinture, musique, danse) et la révolution des ballets russes, comme exemples d'une revitalisation et d'un renouveau de la pensée et de la création : le dialogue des arts mis en scène de manière novatrice, voire provocatrice, pendant la guerre et plus encore dans l'immédiate après-guerre, contribue fortement à rendre possible la crédibilité d'un autre futur que celui du malheur.

Symbolism acted as a pioneer in the artistic movements of the 20th century. The Belle Epoque was followed by the fracture brought about by World War I and by attempts to rebuild the world (with scientific research, various artistic experiments, practice of dialogue between the arts). In addition to contemporary testimonies and commemorations, some artists decided to wipe up the past (Dadaists) and to suggest a new reality (Surrealists). This article reports their activity in Paris (poetry, music, dance) and the revolution of Russian Ballet in order to document the revival and renewal in thought and creativity. The innovative, even provocative dialogue between the arts during the War and even more the immediate post-war period greatly contributed to another remembrance and to the credibility of an alternative future.

\section{Keywords}

Symbolismus $\cdot$ Surrealismus $\cdot$ Krieg $\bullet$ Kunst $\cdot$ Heilung $\bullet$ Resilienz

symbolisme $\bullet$ surréalisme $\cdot$ guerre $\bullet \operatorname{art}(\mathrm{s}) \cdot$ cicatrisation $\bullet$ résilience

symbolism $\bullet$ surrealism $\bullet$ war $\bullet$ arts $\bullet$ cicatrization $\bullet$ resilience

Dans L'Art romantique Baudelaire écrit à propos de « L'OEuvre et la vie de Delacroix » :

[...] quel est donc ce je ne sais quoi de mystérieux que Delacroix, pour la gloire de notre siècle, a mieux traduit qu'aucun autre ? C'est l'invisible, c'est l'impalpable, c'est le rêve, c'est les nerfs, c'est l'âme ; et il a fait cela [...] sans autres moyens que le contour et la couleur ; [...] il l'a fait avec la perfection d'un peintre consommé, avec la rigueur d'un littérateur subtil, avec l'éloquence d'un musicien passionné. C'est, du reste, un des diagnostics de l'état spirituel de notre siècle que les arts aspirent, sinon à se suppléer l'un l'autre, du moins à se prêter réciproquement des forces nouvelles (Baudelaire [1869] $1976: 744)$
Après l'assassinat de l'archiduc François-Ferdinand, héritier de l'empire austro-hongrois, et de son épouse le 28 juin 1914, élément déclencheur d'une guerre dont l'empereur François-Joseph ne voulait pas, c'en est fini de la Belle Époque : les promenades sur les grands boulevards, la fréquentation des cafés, des cabarets, des ateliers et galeries d'arts et des salles de concert n'est plus d'actualité. Et pour la grande bourgeoisie et les aristocrates fortunés, le temps des salons et des saisons, avec l'alternance de la période des spectacles et des réceptions dans les hôtels particuliers et de celle des séjours d'été dans les châteaux à la campagne, dans les villas de la côte normande ou dans les stations thermales ou balnéaires, est révolu : dès le 22 août 1914, 27000 soldats français seront tués en une seule 
journée... Le temps de l'insouciance et de l'optimisme n'est plus : la guerre va prendre une ampleur inouïe et bouleverser les codes traditionnels pour devenir la Première Guerre mondiale. Alors que les premiers belligérants et leurs alliés s'attendaient à une simple redistribution des territoires des Balkans, le bilan du conflit est désastreux, qu'il s'agisse des conditions matérielles de la guerre, du nombre de victimes militaires et civiles, du nombre de blessés aux séquelles irréversibles ou des nouvelles frontières établies par le traité de Versailles (1919), qui redessine l'Europe, les traités de Saint-Germain (1919) et de Trianon (1920), qui procèdent au démantèlement de l'Empire austro-hongrois, et le traité de Sèvres (1920), qui règle le sort de l'Empire ottoman. L'Allemagne, dont le territoire a été fortement réduit, devient une démocratie ainsi que le Royaume-Uni, pour la première fois de leur histoire. Le bilan est encore aggravé par la grippe espagnole qui, à partir de janvier 1918, va infecter un tiers de la population mondiale.

Les traumatismes corporels, psychiques et culturels, qu'ils soient individuels, familiaux, régionaux, nationaux et internationaux, sont divers et multiples... Sortis brutalement d'un monde qui semblait leur promettre bien-être et progrès pour un avenir proche, les êtres humains se retrouvent dans un champ de ruines, internes et externes, et dans un monde qui est à repenser, à reconstruire, pour que l'on puisse le réaccorder, d'une part, à celui qui vient de disparaître tout en restant présent en mémoire, et, d'autre part, à celui qui est à venir, avec la nécessité de garantir la paix et de restaurer la foi dans la prospérité et le bien-vivre. Rétablir des itinéraires de vie pour l'ensemble des survivants, qu'ils soient civils ou militaires, reste une tâche difficile et complexe, ne serait-ce que parce qu'il faut bien admettre que l'impensable n'était pas seulement possible puisqu'il s'est produit et que chacun et chacune sont devant le fait accompli. À tous niveaux de vie et de responsabilité, comment se remettre à avancer et sur quel chemin pour renouer avec le passé et trouver le passage pour un avenir susceptible de prolonger les traces anciennes de la "Belle Époque » sans pour autant renier les années d'une " boucherie héroïque » dont les horreurs ont dépassé dans des proportions insoupçonnées celles que dénonçait Voltaire.

\section{BLESSURES DE GUERRE ET IMPOSSIBLE RETOUR À LA NORMALITÉ}

Dans la mesure où l'ampleur des traumatismes relève de l'incompréhensible, la première des nécessités est de pouvoir en parler, de parvenir à dire et raconter ce qui s'est passé, pour en trouver le pourquoi et le comment, afin que ce qui a détruit tant et tant de vies, à tous points de vue, soit effectivement intégré à l'histoire, non seulement de chacun, mais à celle de toutes les communautés auxquelles tout individu appartient, et plus généralement à celle du " monde ". Toute reconstruction passe par des manifestations et des récits de témoignage. Les spécialistes de sciences humaines, historiens, sociologues et psychologues, ont établi des méthodes et des protocoles spécifiques pour que la « vérité » événementielle soit assumée, le plus objectivement possible, pour les uns par la certification de la documentation réunie et par la prise en compte des déterminations et des attentes sociales, et pour les autres, plus subjectivement, selon les troubles affectifs, psychologiques et comportementaux résultant des traumatismes engendrés.

Les guerres n'ont certes pas manqué dans l'histoire tourmentée de l'Europe et dans les relations entre provinces, régions, royaumes, pays, et nations. Les artistes, quelle que soit leur discipline de création, ont toujours été invités à participer aux chants, aux cérémonies et aux commémorations concernant les victimes et les soldats. Mais l'actualisation des codes établis concernant la guerre et ses représentations, la célébration des héros, les hommages aux victimes, dans leurs formes traditionnelles, qui sera pratiquée dans les années qui vont suivre l'immédiate après-guerre, ne suffiront pas à réduire les terribles fractures et les profondes ruptures engendrées par la Grande Guerre. Comme il est coutume, de très nombreux monuments aux morts furent dressés dans les villes et villages, particulièrement en France et en Allemagne, qui comptaient un nombre écrasant de victimes. Pour autant les blessures restaient ouvertes, et pas seulement parce que les ratifications des traités laissaient beaucoup d'insatisfactions et d'amertumes. En France et en Belgique la cicatrisation de la terre est aujourd'hui toujours en cours; sous la protection intemporelle des mausolées et des ossuaires, les démineurs restent mobilisés : un obus sur quatre n'a pas explosé, et même quatre sur cinq lorsqu'ils tombaient dans l'eau ou en zone humide. Par ailleurs certains obus pouvaient s'enfoncer jusqu'à trente mètres de profondeur selon la nature du terrain. Quasiment toutes les familles ont dû faire leur deuil d'un ou de plusieurs proches, s'occuper d'un invalide ou pleurer un disparu... Et l'histoire récente comme l'histoire ancienne, malgré leurs vestiges, leurs monuments et leurs multiples traces et évocations, malgré le florissement des philosophies sociales et tout ce qui constitue ordinairement des recours possibles pour revenir à une hypothétique normalité, ne fournissent pas de modèles de pensée et de représentation susceptibles de correspondre au rétablissement de la vie dans ses aspects positifs, - matériels, émotionnels et spirituels.

Une fois épuisées les " postures » et les « recettes » léguées par la tradition et les institutions, que reste-t-il sinon autant que possible le dire, le faire et le vivre autrement? En passer par exemple par la « table rase », et faire comme si l'on pouvait repartir à zéro, ainsi que le suggère le dadaïsme de Tristan Tzara, étape préalable pour accéder à la surréalité, revenir au pur langage dégagé de tout ce qui masque et corrompt la réalité et la vérité des êtres et des choses :

Au soir de la folie, nu et clair

L'espace entre les choses a la forme de mes paroles,

La forme des paroles d'un inconnu,

D'un vagabond qui dénoue la ceinture de sa gorge

Et qui prend les échos au lasso.

(Éluard 1968 : 175) 
C'est ainsi que les institutions scolaires et universitaires présentent généralement la naissance du surréalisme. En réalité, c'est en grande partie dans le tourbillon des mouvements artistiques et littéraires initiés par le symbolisme que se renoue le fil entre l'ancien monde englouti et le nouveau monde qui va peut-être presque naître.

\section{L'HÉRITAGE DU SYMBOLISME À PARTIR DE BAUDELAIRE}

Durant les années d'avant-guerre, l'effervescence dans le monde des intellectuels, des artistes, de toute la population qu'on appelle volontiers « la bohême », tout à la fois mondaine et frondeuse, est grande, à Paris certes, mais également dans les capitales européennes. D'abord décadentistes puis symbolistes, les adeptes de la modernité d'alors ont souvent plusieurs cordes à leur arc et pratiquent généralement avec bonheur poésie, arts plastiques, et musique, non sans de multiples variations, explorations, et provocations en tous genres, car ils contestent les codes établis et les académies, d'où leur appellation d'origine, " décadentistes ", par antiphrase. Les Romantismes allemand et anglais avaient déjà proposé de nouvelles perspectives, et Baudelaire apparaît comme le père spirituel du symbolisme qui bouleversera peu à peu la pensée créatrice des artistes et leurs œuvres dans la richesse de leur diversité, de la fin du XIXème siècle aux confins du XXème. Alors que traditionnellement la création artistique reposait sur l'articulation du couple " dire " et " représenter » dans les différents langages de spécialité propres aux arts, l'on est passé au « suggérer » et à l'« évoquer », en ayant recours aux multiples possibilités d'expression formelle propres à chaque langage artistique, et parfois même à de singuliers mélanges, avec un rapport au monde qui n'est plus celui de l'intellect -, raison et vérité conceptuelle -, mais celui d'une perception correspondante du sensible, du matériel et du spirituel, avec le recours à l'imagination, "la reine des facultés » selon Baudelaire, qui parle de son art en termes de " sorcellerie évocatoire » et de « magie suggestive ». Qu'il s'agisse de microcosme ou de macrocosme, tout est à déchiffrer, et la connaissance de soi comme de tout ce qui l'entoure passe par l'accord de la vie sensible avec la vie spirituelle, - dont les correspondances de Fourier, de Swedenborg et de Toussenel, revisitées par Nerval et Baudelaire : celles qui sont horizontales, - les synesthésies -, et celles qui sont verticales, - du terrestre au céleste, de ce qui relève des sens à ce qui relève de l'esprit.

Il est vrai aussi que les progrès technologiques ont incité les créateurs artistiques à revoir leurs codes et leurs manières de faire et de penser. Ainsi les dioramas et la photographie ont conduit à repenser ce qui relevait de la représentation. En peinture, le statut des portraits, des natures mortes, des représentations animales et florales se modifie. L'invention des tubes de peinture permet aux artistes peintres d'exercer leur art à l'extérieur : les lumières changent, le paysage sort des conventions de fonds de tableaux et devient un sujet à part entière...
Bref, de 1880 à 1920, dans le flou immense de l'intense créativité artistique de l'époque que certains regroupent un peu abusivement en "symbolisme(s) ", quitte à le décomposer à plus ou moins juste titre en étiquettes d' "écoles ", tant nous aimons figer les choses en mouvement pour essayer de mieux les comprendre, naissent et se développent l'impressionnisme (dès 1860) et le postimpressionnisme, le pointillisme et le divisionnisme, le fauvisme, le nabisme, le préraphaélisme, le synthétisme, le primitivisme, l'orphisme, l'expressionnisme et son corollaire abstrait, le futurisme italien, l'Art déco, l'Art nouveau, l'art abstrait, le nouveau réalisme, le cubisme, et le frémissement du surréalisme à venir. À l'inverse d'autres oublient le "Symbolisme », dont l'initiative est trop imprécise, entre France, Belgique, Russie etc., sous des prête-noms célèbres, mais sans véritables chefs de file clairement identifiés et revendiqués... L'héritage de Baudelaire ne cessera cependant d'être exploité et continue à l'être encore aujourd'hui, bien au-delà des frontières de l'Hexagone et de la Belgique.

\section{ORIENTATIONSPLURIDISCIPLINAIRESDELA MODERNITÉ POST-BAUDELAIRIENNE}

Dans le fouillis et la diversité de la modernité artistique post-baudelairienne, se dessinent cependant des constantes, dont la rébellion et la révolte contre les ordres établis et la volonté de faire authentiquement dialoguer les langages artistiques entre eux, non sans remettre en question leurs définitions et leurs fonctions discursives. Certes les convictions idéologiques et les croyances des uns et des autres ne sont pas nécessairement identiques, voisines ou compatibles, d'autant que tous n'ont pas la même conception de la " réalité » et du « réalisme ", puisque pour tous ce qui est en question c'est justement la relation à la réalité telle qu'elle est déterminée par les habitudes de discours, de représentations et de valeurs qui lui sont attachées.

Ce que l'on appelle ordinairement le dialogue des arts est au cœur des propositions des symbolistes. Non seulement il ne s'agit pas de décrire mais d'évoquer le monde en prospectant les images et leurs similitudes tout en prenant en compte les états d'âme, les atmosphères et les idées abstraites, et il s'agit aussi de rendre à chaque langage artistique toutes ses possibilités d' " impression " et d' " expression », y compris en faisant en sorte qu'ils « se prêtent réciproquement des forces nouvelles ".

Outre les champs de ruines, les victimes, les blessés, les handicapés et les gueules cassées, les tranchées ouvertes, les sites naturels bafoués et empoisonnés, la Grande Guerre laisse un chaos éthique, esthétique, sociologique, idéologique et philosophique. Avec des nuances et des différences selon les lieux, tout ou presque est à reconstruire pour renouer le fil de la vie, celui des vivants et des survivants, celui de la famille, de la communauté, celui du cadre de vie, de l'espace et de l'environnement, celui du temps aussi, du rythme des jours et des semaines, des mois, des saisons et des 
années, du rapport à soi, aux autres, aux disparitions et à ce monde pas encore nouveau devenu si difficile à ressentir et à comprendre.

Pour que la cicatrisation parvienne à se faire, il faut d'abord panser les blessures, exorciser la douleur et le contraste si affligeant des souvenirs heureux et des horribles souffrances qui leur ont succédé avec les angoisses et les incertitudes du présent, et pouvoir enfin se projeter dans un véritable avenir... Le nécessaire travail de résilience qui permet aux individualités et à leur communauté de se réadapter à la vie en parvenant à intégrer à la réalité des événements qui ont dépassé leur entendement, au point de ne plus reconnaître le monde qui était le leur et à ne plus se reconnaître eux-mêmes, passe par une catharsis d'autant plus difficile que les langages, - leurs normalités du dire et leurs conventions discursives -, sont eux aussi en crise. Et cependant il faut parvenir à sortir de la stupéfaction, de la sidération et de l'hébétude, même si parfois elles sont liées à une forme de fascination analogue à celle que peut exercer le crime sur la sensibilité populaire, et parvenir à réharmoniser les émotions et les pensées, en sachant qu'il en va de "l'âme " comme du " corps » : même lorsque les douleurs physiques ont disparu, les cicatrices restent et témoignent de l'impossibilité d'un véritable oubli, d'autant que la tentation ou le " goût du néant " menace. Et même la douce Nuit qui marche, chère au poète, n'est que le linceul passager d'un sommeil qui n'est plus réparateur puisque ne s'inscrivant que dans le temps du recueillement.

Les legs des traditions institutionnelles, coutumières ou cérémonielles, qui rythment les années de l'après Grande Guerre, par nécessité et par habitude, tentent bien de témoigner des combats et des horreurs qui ont fait tant de dégâts en les inscrivant dans les paysages familiers des places de villes et de villages, des parcs et des cimetières, des musées et salles d'exposition aussi - sous forme de monuments aux morts, de mausolées, de statues, de plaques de mémoire et de tableaux -, mais ils ne peuvent suffire à recréer un univers de paroles, de discours et de représentations propres à redonner espoir, confiance et foi dans une vie nouvelle et authentiquement « revitalisée ».

Félix Valloton, peintre suisse formé à l'Académie Julian à Paris, inhumé au cimetière Montparnasse, fit partie d'une mission artistique sur le front en 1917. En 1915-1916 il avait produit une série de bois gravés intitulée C'est la guerre ! De sa mission il rapporta des toiles représentant un univers asphyxié sous forme de fragments recomposés et dans lesquelles on entend le grondement lointain du canon, dont un Verdun oppressant, tout à la fois lumineux et abstrait, aujourd'hui exposé au Musée des Armées. Après la guerre les paysages qu'il produit rendent compte et nous parlent d'une nature déshumanisée aux étranges couchers de soleil qui semblent contemplés par un spectateur indiscret, revenu du royaume des morts pour essayer de se persuader de sa propre existence. Félix Vallotton avait publié en 1907 un livre intitulé La vie meurtrière et son œuvre picturale s'inscrit déjà dans celle d'Edward Hopper et dans les productions du Pop
Art tout en ayant été influencée par Paul Gauguin, les Nabis, et par Édouard Vuillard. Sa mission sur le front le conduira à affirmer dans un essai paru en 1917 que « Peindre la guerre aujourd'hui ce n'est plus peindre des tableaux de bataille » et qu'aucune peinture ne peut traduire le fond épouvantable du conflit. La dimension décorative de ses toiles de guerre, qu'il reconnaît, témoigne aussi d'une forme de fascination mêlée à la peur. Dans les dernières lignes de son Journal il écrit : "La Vie est une fumée, on se débat, on s'illusionne, on s'accroche à des fantômes qui cèdent sous la main, et sa mort est là », en ajoutant cependant ce correctif : « II reste la peinture heureusement. »

De nombreux artistes, de part et d'autre, ont été mobilisés. Certains ont été tués, d'autres blessés, quelques-uns ont été écartés du front pour raisons de santé, d'âge ou de charges familiales. D'autres n'étaient pas mobilisables. Pour autant, les grands centres intellectuels et artistiques que sont Berlin, Vienne et Paris ne restent pas inactifs, tout particulièrement dans la capitale française, dans le quartier Montparnasse, la butte Montmartre et à Puteaux. Ainsi Pablo Picasso, Amedeo Modigliani et Leonardo Foujita, non mobilisables, se retrouvent-ils dans les cafés de Montparnasse ou chez Marie Vassilieff ${ }^{1}$. En 1917 de nombreux écrivains et artistes, français et étrangers, rentrent à Paris, à Montparnasse le plus souvent, vivants, mais souvent blessés dans leur chair et à jamais dans leur être : Hans-Jean Arp, Georges Braque, Blaise Cendrars, Jean Cocteau, André Derain, Moïse Kisling, Fernand Léger, André Salmon, Ossip Zadkine et la liste est loin d'être complète... Leur participation ou leur engagement dans la Grande Guerre les conduisent à la même conclusion que celle de Vallotton : on ne peut plus « dire " et « représenter » comme avant, même s'ils seront nombreux à vouloir témoigner de ce qu'ils ont vu et vécu, et à se heurter à la difficulté du témoignage. II serait cependant erroné de considérer que la modernité du XXème siècle est directement et exclusivement issue de l'après-guerre 14-18. Plusieurs écrivains et artistes de renom n'éprouveront par exemple le besoin de parler de la guerre qu'ils ont vécue que lorsque la menace d'une nouvelle hécatombe mondiale se profilera.

D'une part les propositions du symbolisme et des symbolistes dans leur diversité se sont répandues à la fin du XIXème siècle dans toute l'Europe, grâce notamment au « groupe des $\mathrm{XX}$ » de Bruxelles, et d'autre part les innovations et les bouleversements techniques, scientifiques et théoriques qui se développeront durant le $X X X^{\text {ème }}$ siècle sont déjà initiés avant 1914. Le train express, le métropolitain, le téléphone, l'automobile et l'avion se perfectionnent et transforment en profondeur la relation humaine au temps et à l'espace.

Grâce à de nouveaux outils, l'exploration macroscopique et microscopique du monde progresse et, avec Einstein, Becquerel, Pierre et Marie Curie, de nouvelles interrogations concernant la matière surgissent. Même si les travaux du

1 Peintre russe d'origine ukrainienne, surnommée « la cigale des steppes », qui a fermé en 1914 son académie de Montparnasse afin d'ouvrir une cantine pour artistes désargentés, laquelle devint rapidement un lieu de rendez-vous pour nombre d'artistes aujourd'hui connus et reconnus. 
docteur Freud sont déjà en chantier en 1890, on peut dater ses découvertes concernant l'inconscient du 21 septembre 1897 lorsqu'il renonce officiellement à la théorie de la séduction pour celle du fantasme. Ce foisonnement scientifique et technologique, voire « mécaniste », est abondamment relayé et diffusé par les journaux et les revues qui, à l'époque, intéressent vivement les artistes, écrivains, peintres, poètes et compositeurs. Les métalangages ou langues de spécialités s'interpénètrent et les expérimentations se multiplient : d'une certaine façon les arts et les sciences contribuent au développement de nouveaux modes de pensée et de représentation, dans lesquels la connaissance sensible l'emporte sur le rationalisme, alors que l'« imagination » reste fondamentalement "la reine des facultés " tout en se nourrissant des inventions et des découvertes scientifiques : la peinture de Paul Signac en témoigne pour ce qui est de la lumière ; celles de Fernand Léger et de Marcel Gromaire, avec La partie de cartes et La guerre par exemple, montrent la déshumanisation mécaniste des soldats... Avant et pendant la Grande Guerre, Paris est la capitale mondiale des arts et de l'effervescence intellectuelle et c'est à Paris que naissent les deux grands courants que sont au XXème siècle le fauvisme et le cubisme et qu'est prospecté le primitivisme (l'art nègre par exemple) dans le souci de retrouver la vérité des langages originels. À Puteaux, la "Section d'Or » qui soutient le cubisme cherche à atteindre l'harmonie en utilisant le nombre d'or, ce qui nous ramène à l'antiquité grecque et à Nerval, et nous conduit aussi à Le Corbusier... Le langage du fauvisme a donné la primauté absolue à la couleur, débarrassée de sa fonction d'imitation de la nature. La couleur pure vaut par ce qu'elle peut révéler ou libérer d'émotion ou de sensation, et dans ces temps de guerre, si l'on s'aventure volontiers à briser les lignes, on n'hésite pas à faire éclater les couleurs. En ce sens le fauvisme s'accorde avec l'expressionnisme du mouvement « Die Brücke » né à Dresde en 1905 à l'initiative de quatre amis architectes et celui du mouvement « Der blaue Reiter » fondé à Munich en 1911 par Vassily Kandinsky dans le sillage de «La Nouvelle association des artistes munichois » qui regroupait des artistes allant du néo-impressionnisme à l'Art nouveau et dont il fut président. En 1907-1908 Kandinsky avait séjourné à Paris... Par la suite il évoluera vers l'art abstrait dont il est considéré comme le fondateur : la forme et la couleur auront pour mission de donner le sens « d'une représentation graphique d'un état d'âme ». Kees van Dongen, d'origine néerlandaise qui s'est lié d'amitié avec Maurice de Vlaminck et Henri Matisse et vit à Paris fit le lien avec les expressionnistes allemands. Ce qui unit les artistes de ces mouvements c'est à la fois le primat accordé à la couleur pure et l'importance relative donnée aux préoccupations formelles. Les cubistes en particulier qui travaillent à partir de notions mathématiques sur des cônes, des cylindres et des sphères pour représenter un « réel recomposé "s'inscrivent dans un certain formalisme que récusent les expressionnistes. Ce qui les unit c'est aussi le souci de s'intéresser davantage à ce que l'objet peut faire ressentir, éprouver et suggérer qu'à sa représentation proprement dite, ainsi que la diversité des " éléments de langage " susceptibles d'être utilisés, d'où les collages, les graphismes et parfois le choix des matériaux etc... " Le dialogue des arts » est largement ouvert, même si les principes d'unité et de cohérence continuent à être recherchés malgré des apparences parfois plus que surprenantes. Les avant-gardes de l'époque n'ont pas échappé aux tohu-bohu, aux chahuts et aux critiques véhémentes ou lapidaires.

En 1916 l'immense atelier que le peintre suisse Émile Lejeune met à disposition de ses amis artistes, - les lieux disponibles sont rares dans le contexte de la guerre devient la salle Huygens, salle d'expositions et de spectacles, sous les estampilles des sociétés "Lyre et Palette » et "Palette et Musique », et véritable vivier de la création artistique où se retrouvent poètes, peintres, littérateurs, sculpteurs, musiciens, collectionneurs et amateurs d'art jusqu'au début des années vingt. Le groupe des Six, qui pour I'heure répond au vocable « Nouveaux jeunes »-, Georges Auric, Louis Durey, Arthur Honegger, Darius Milhaud, Francis Poulenc et Germaine Tailleferre -, y réalise des performances. Et l'on est déjà au-delà de l'impressionnisme et du wagnérisme...

Le 18 mai 1917, un projet de spectacle « pluridisciplinaire » auquel tenaient tout particulièrement Jean Cocteau et Erik Satie trouve son aboutissement au théâtre du Châtelet : il s'agit de Parade. C'est en 1910 que Jean Cocteau, qui a déjà travaillé avec Serge de Diaghilev pour d'autres ballets, lui présente le projet, inspiré d'une toile de Georges Seurat, Parade de Cirque. Diaghilev l'inscrit dans les programmes « modernistes " de ses Ballets russes. L'affiche de présentation en mai 1917 porte le titre de "Ballet réaliste » et si l'on en croit la presse, les témoignages d'époque et la réflexion du musicien, « Ce fut un beau scandale »! Le ballet dure une demi-heure ; le texte est de Cocteau, la musique de Satie, la chorégraphie de Léonide Massine et la scénographie, - décors, costumes et rideau de scène -, de Pablo Picasso. Le texte du programme rédigé par Apollinaire et déjà publié le 11 mai dans le journal Excelsior, - quotidien illustré, qui parut de 1910 à 1940, traitant de l'information au jour le jour en privilégiant l'illustration photographique -, célèbre surtout Picasso et Massine pour avoir réalisé une « alliance nouvelle », celle de la peinture et de la danse, de la plastique et de la mimique, « signe évident de l'avènement d'un art plus complet ». Apollinaire poursuit :

De cette alliance nouvelle, car jusqu'ici les décors et les costumes d'une part, la chorégraphie d'autre part, n'avaient entre eux qu'un lien factice, il est résulté une sorte de sur-réalisme où je vois le départ d'une série de manifestations de cet Esprit Nouveau, qui, trouvant aujourd'hui l'occasion de se montrer, ne manquera pas de séduire l'élite et se promet de modifier de fond en comble, dans l'allégresse universelle, les arts et les mœurs, car le bon sens veut qu'ils soient au moins à la hauteur des progrès scientifiques et industriels ${ }^{2}$.

2 L'Esprit Nouveau est par ailleurs une revue fondée par Le Corbusier et Amédée Ozenfant en 1920, dirigée par Paul Dermée, écrivain, poète et critique littéraire belge, qui a collaboré en 1916 à la revue d'avant-garde SIC et en 1917 à celle de Pierre Reverdy Nord-Sud. 
Dans l'Excelsior du 18 mai 1917, Jean Cocteau insiste sur la portée fondatrice de Parade :

Parade groupe le premier orchestre d'Erik Satie, le premier décor de Picasso, les premières chorégraphies cubistes de Léonide Massine et le premier essai pour un poète de s'exprimer sans paroles.

Et si le ballet renvoie à " une sorte de sur-réalisme " c'est parce que la visée était de représenter « quelque chose de plus réel que la réalité », d'où le titre de présentation « Ballet réaliste ». Même s'il est possible que les mots de " sur-réalité » et de " sur-réalisme » aient été prononcés et peut-être suggérés à Guillaume Apollinaire par Jean Cocteau et Picasso, il est certain que le vocable, réinterprété par André Breton et devenu célèbre quelques années plus tard, a été " créé ", au sens théâtral du terme, par l'auteur des Mamelles de Tirésias, drame en deux actes avec prologue sous-titré "drame surréaliste ", ainsi que le confirme une lettre de mars 1917 envoyée à Paul Dermée :

Tout bien examiné, je crois qu'il vaut mieux adopter surréalisme que surnaturalisme que j'avais d'abord employé. Surréalisme n'est pas encore dans les dictionnaires et il sera plus commode à manier que surnaturalisme déjà utilisé par MM. les philosophes.

\section{ALLIANCE DES ARTS ET CICATRISATIONS}

L'année 1917 fut particulièrement horrible. Mais paradoxalement les deux représentations que nous avons évoquées constituent non seulement des événements majeurs pour les développements artistiques, éthiques et politiques qui gagnent toute l'Europe et constitueront des modes de cicatrisation dans la mesure où se dessinent pour les individus, les communautés et les institutions de nouvelles modalités de discours et de pensée pouvant restaurer l' " espoir » d'un avenir « autre " et produire de nouvelles définitions de l'« engagement », des rapports sociaux et des relations au monde, et donc de nouveaux modes de représentations de soi et de la réalité environnante...

Pourquoi nous être attaché à rappeler ce que fut l'apport du foisonnement symboliste et à montrer comment il avait infiltré profondément le nouveau et le renouveau auxquels aspiraient non seulement "la bohême " artistique, mais bien des élites intellectuelles et scientifiques en Europe, si ce n'est parce qu'alors même que se produit le cataclysme d'un conflit hors normes, au plus fort de la tourmente, fructifient paradoxalement les germes de la reconstruction et de la résilience. Apollinaire, voyant les drapeaux installés pour célébrer le 14 Juillet, déclarait déjà le 13 juillet 1909 dans un "Poème lu au mariage d'André Salmon » : « [...] je ne me suis pas dit [...] on renouvelle le monde en reprenant la Bastille. [...] Je sais que seuls le renouvellent ceux qui sont fondés en poésie. » Et l'on songe en écho à cette affirmation de Baudelaire : «La poésie est ce qu'il y a de plus réel, c'est ce qui n'est complètement vrai que dans un autre monde. ${ }^{3}$ » Les conditions de vie en temps de guerre ont contraint les " créateurs ", quels que soient leur langage et leur domaine d'activité et de production de trouver des lieux dédiés, le plus souvent privés, où se rencontrer, échanger et travailler ensemble. La guerre reste présente dans presque toutes les œuvres créées, avec les variations " discursives », éthiques et esthétiques que nous avons évoquées. Ainsi pour la représentation du 24 juin 1917, Apollinaire ajoute aux deux actes des Mamelles de Tirésias rédigés dès 1903 un prologue et une scène finale, qui accentuent l'antimilitarisme et ce qu'on peut appeler le féminisme du « drame surréaliste ». Bien évidemment, durant la Grande Guerre, notamment en littérature et en peinture, l'introspection et le rêve cèdent aussi le pas au souvenir oppressant des tranchées, aux horreurs et aux misères qui marquent le quotidien et s'inscrivent dans l'absurdité présente du monde : les expressionnistes allemands et autrichiens en témoignent tout particulièrement. Mais l'interpénétration du réel ordinaire et du rêve de l'inconscient permettent aux artistes de prolonger les nombreux acquis issus des mouvances symbolistes. Marc Chagall qui s'inscrit dans l'expressionnisme travaille la couleur et déforme la réalité pour susciter l'émotion et inciter à voir et à penser autrement. De fait, à chaque discipline artistique correspond un langage spécifique, dans sa matérialité et dans ses contraintes techniques, voire technologiques, qui n'ont cessé d'évoluer dans le temps comme c'est le cas pour les langues naturelles et même les langues artificielles. Les possibilités discursives de chaque langage artistique, au premier chef la littérature, sont à la fois contraintes et infinies, d'autant que chaque " créateur " s'empare des possibilités langagières de sa discipline en les actualisant de manière singulière. Non seulement chaque " créateur » inscrit son œuvre dans une actualisation spécifique de la langue artistique qui est la sienne, mais chaque objet produit est un discours autonome qui fait partie des variations discursives prospectées par l'artiste lui-même et qui font en quelque sorte entendre sa " voix ", chaque fois, par exemple, quand son regard nous « parle » lorsque nous regardons l'une de ses toiles, s'il est artiste-peintre.

Au-delà des chahuts, des polémiques et des arrières plans politico-idéologiques provoquées par les représentations de Parade et des Mamelles de Tirésias en 1917, nous retiendrons que les contraintes de la guerre et que les multiples avancées des décennies qui ont précédé le premier conflit mondial ont incité les artistes et certaines élites à se regrouper et à confronter les possibilités discursives de leurs langages de spécialité pour produire des discours dans lesquels, d'une part, la pluridisciplinarité n'est pas « factice » et, d'autre part, parler de tout sur tous les tons est toujours possible. Même si Philippe Soupault, qui était souffleur lors de la première des Mamelles de Tirésias s'est

3 Projet d'article concernant le peintre « réaliste moderne » Gustave Courbet, intitulé Puisque réalisme il y a. 
dit par la suite « indigné par le décalage entre ces farces [Parade et le " drame surréaliste "] et l'horreur vécue par les soldats ", Jean Cocteau lui avait déjà répondu dans les colonnes de l'Excelsior le 18 mai 1917, sous le titre «Avant-Parade » :

[...] La force de la France éclate de toutes parts sous le tonnerre d'avril. Impossible d'interrompre ce besoin de créer, ce jaillissement, cette écume de verdure irrésistible qui s'oppose au lourd esthétisme germain. Nous souhaitons que le public considère Parade comme une œuvre qui cache des poésies sous la grosse enveloppe du Guignol. Le rire est de chez nous ; il importe qu'on le soutienne et qu'on le ressuscite même aux heures les plus graves. C'est une arme trop latine pour qu'on la néglige.

II ne s'agit plus seulement de « redonner un sens plus pur aux mots de la tribu ! ", comme le proposait Mallarmé, mais de bousculer sans interdit l'ensemble des codes établis, quels que soient la discipline, le domaine et le langage de création : genres, tonalité, sémantique, modes d'énonciation, registres, syntaxe, rythme etc... L'heure est à l'expérimentation de tous les possibles, sans barrière entre l'extravagance, la fantaisie et la gravité des " sujets ", l'objectif étant de libérer la parole pour un discours à plusieurs voix, poétique, pictural, musical et apparemment loufoque, susceptible de faire voir et entendre la « vraie » réalité contre celle qui ne cesse d'être convenue, trompeuse, hypocrite et donc susceptible d'incompréhensions, de polémiques et de conflits. Bref, une entrée ou un retour dans cette "vitalité » dont parlait Baudelaire.

Apollinaire, dans une conférence sur L'Esprit Nouveau et les poètes, retranscrite, est revenu sur cet « Esprit nouveau » et son vent de liberté nouvelle :

II ne faut pas oublier qu'il est peut-être plus dangereux pour une nation de se laisser conquérir intellectuellement que par les armes. C'est pourquoi l'esprit nouveau se réclame avant tout de l'ordre et du devoir qui sont les grandes qualités classiques par quoi se manifeste le plus hautement l'esprit français, et il leur adjoint la liberté. Cette liberté et cet ordre qui se confondent dans l'esprit nouveau sont sa caractéristique et sa force. (Apollinaire [1917] 1991 : 96)

...peut-être pour préciser la profondeur " sur-réelle » de son drame qui s'inscrivait dans le vivre d'actualité à son époque - la guerre, le féminisme, la natalité, la presse...-sous l'apparence d'une « folie poétique » produite par une imagination délirante dans une mise en scène fantaisiste. La bouffonnerie et le rire n'excluent pas le tragique engendré par la guerre et peuvent constituer une forme salutaire d'exutoire dans un processus cathartique : le rire correspond à une gaieté moderne, profonde et tragique.

Au-delà de l'importance attribuée au comique, à la bouffonnerie et à la clownerie aux côtés de l'ironie et de la satire, ce sont les « forces nouvelles » que se prêtent « réciproquement les arts » qui sont à souligner. Assurément « Le Dialogue des Arts » existe depuis longtemps, comme l'a montré Gérard
Denizeau dans son livre au titre éponyme sous-titré « architecture peinture sculpture littérature musique » auquel il faudrait ajouter "cinéma ". "Le livre de dialogue », selon l'expression d'Yves Peyré, est pratiqué à la fin du XIXème siècle et tout au long du $X X^{\text {ème }}$, particulièrement en ce qui concerne la peinture et la poésie (Peyré 2008 ; Bergez 2008). Et depuis les années 1871, la « Mélodie » française est venue concurrencer le « Lied » germanique. En février ou mars 1917, Claude Debussy compose une pièce pour piano longtemps restée inédite, qu'il intitule d'un alexandrin de Baudelaire extrait du « Balcon », « Les Soirs illuminés par l'ardeur du charbon » (Baudelaire [1869] 1976 : 37), qu'il offre en remerciement à son pourvoyeur de charbon. Maurice Ravel écrira entre 1929 et 1931 un Concerto pour la main gauche à la " véhémence tragique " pour le pianiste autrichien Paul Wittgenstein qui avait perdu le bras droit au cours de la guerre. La première de Parade avait été donnée au bénéfice des Ardennais, société philanthropique consacrée aux sinistrés de l'Est... La guerre en 1917 s'inscrit sur l'ensemble des fonds de la création artistique.

Ajoutons encore que Les Mamelles de Tirésias ont poursuivi et poursuivent encore une carrière internationale : le drame fut joué à Prague et en Italie dès les années 1926-27, le texte fut repris par Francis Poulenc dans un opéra-bouffe créé en 1947, traduit et créé en anglais avec succès aux États-Unis dans les années cinquante, comme en témoigne une lettre de Léonard Bernstein, et en allemand, à Bâle, en 1957.

Certes l'héritage du symbolisme romantique, dont le « programme » peut se résumer à cette phrase de Mme de Staël :

[...] il faut, pour concevoir la vraie grandeur de la poésie lyrique, errer par la rêverie dans les régions éthérées, oublier la terre en écoutant l'harmonie céleste, et considérer l'univers entier comme un symbole des émotions de l'âme.

...n'est plus d'actualité dans les années de guerre et d'après-guerre. En revanche les créateurs d'art apprennent à faire front en unissant leurs forces et en mutualisant en quelque sorte leurs possibilités d'expression et celles du ou des langages qu'ils maîtrisent ou qu'ils comprennent ${ }^{4}$ : ils ne cessent d'apprendre les uns les autres de nouvelles façons de sentir et de percevoir, de parler et de faire voir. En ce sens parler du « dialogue des arts » demande à être précisé, dans la mesure où la démarche ne relève pas d'une pensée alternative. Qu'il s'agisse d'harmonie ou de disharmonie, les phases d'élaboration d'œuvres communes se caractérisant par des discussions voire des disputes et des affrontements, le discours produit se doit d'être à l'unisson, c'est-à-dire avoir son unité et sa cohérence, son " ordre » si l'on préfère pour reprendre le mot d'Apollinaire, tout en affichant sa « liberté ». S'il y a dialogue, il reste interne, et contribue au discours qui est produit. Pour être plus explicite, nous pouvons utiliser l'analogie de la musique orchestrale : en définitive la voix

4 Qui se souvient aujourd'hui que Pablo Picasso a écrit en 1941 une pièce de théâtre en six actes intitulée Le Désir attrapé par la queue? 
que l'on entend est celle de l'orchestre, composée des multiples voix spécifiques des instruments accordés lors des répétitions et à fortiori des concerts sous la houlette d'une ou d'un chef d'orchestre selon sa lecture-écoute de la partition. Chaque musicien maîtrise à sa manière les possibilités d'expressivité de son instrument mais possède suffisamment de formation et de culture musicale pour comprendre celles des autres instruments. Par ailleurs les inventions techniques et technologiques qui concernent les différentes disciplines artistiques peuvent s'influencer réciproquement. La création littéraire par exemple peut construire des représentations visuelles et/ou auditives avec des mots, des syntagmes, des constructions de phrases, disposés de multiples façons : par petites touches juxtaposées à la manière de la peinture impressionniste, par une approche linguistique globale sous forme d'ekphrasis ou d'hypotypose, par utilisation des possibilités rythmiques et prosodiques de la langue comme dans la "répétition obstinée " en musique par exemple, voire sur les modèles rhapsodiques ou même sur une distribution calligrammatique... De plus les délimitations des genres, dans l'ensemble des disciplines artistiques, ne cessent d'être contestées et de se reconfigurer, quitte à se mélanger. Et par ailleurs, les variations des modes de " lecture " sont tributaires des variations énonciatives qui donnent souvent à " lire » le même " objet d'art » selon plusieurs lignes discursives à entendre simultanément, d'autant que l'ambition de la génération moderne est de rendre compte d'une prise de connaissance de la réalité qui est à la fois celle du sensuel et du sensible et celle du spirituel et, peut-être, du conceptuel. Le créateur d'art inscrit toujours l'« objet " qu'il produit dans un discours spécifique dont il est le premier " interlocuteur ": " Je " est un autre ! écrit le poète Arthur Rimbaud dans sa lettre à Paul Demeny datée du 15 mai 1871. Bien qu'il n'y ait pas de réversibilité, les « interlocuteurs » suivants seront les « lecteurs » à venir, puisqu'ils interpréteront l'« objet " en fonction de ce qu'ils sont, de ce qu'ils ressentent et de ce qu'est l'« encyclopédie » de référence qui leur est propre. On a beaucoup parlé dans le sillage du structuralisme de grammaire de langue, de grammaire de texte et d'agrammaticalité notamment à propos de poésie. Mais force est de constater qu'un poème n'est pas une construction linguistique actualisant positivement ou négativement des modèles grammaticaux dans des modèles éventuellement strophiques et métriques... La pertinence du poème s'élabore strictement dans le discours qui le construit, à partir d'une sensation, d'une émotion, d'un ressenti particulier que le poète désire transmettre. Ses choix ne se font pas vraiment au mot à mot, à telle ou telle figure, même si cela se produit occasionnellement, mais selon la dynamique même du discours qui se crée en se faisant, à tous les niveaux de sa matérialité, quitte à y revenir pour faire des corrections. Certains artistes reviennent sans cesse sur les " objets " qu'ils ont produits pour les corriger et les améliorer, et qui ne deviennent définitifs que lorsque la mort ne permet plus de continuer à les entretenir...Il serait erroné de penser que l'objet d'art se définit exclusivement comme la réalisation concrète d'une intention créative.

\section{CONCLUSION}

Nous sommes en partie responsable du fait que la dimension anthropologique de la stylistique est revenue dans le champ des disciplines artistiques depuis plusieurs années, et nous continuons à penser qu'elle est une méthode d'analyse critique qui permet en diachronie et en synchronie de préciser la pertinence des discours que les créateurs nous tiennent par la médiation des objets d'art dans lesquels ils sont toujours présents. Et si les œuvres d'art peuvent contribuer à nous remettre dans la vie c'est aussi parce qu'elles ne cessent de nous faire voir et entendre des choses que nous ne savons pas.

Sous cet angle, la Grande Guerre a provoqué une sorte d'excitation, d'exacerbation et d'urgence dans le monde de la création artistique comme si toute l'effervescence des années d'avant-guerre avait pour aboutissement de parvenir à faire voir à tout prix au milieu de l'angoissante et mortelle tourmente et de ses décombres « la vie en beau » selon l'expression de Baudelaire dans "Le mauvais Vitrier": changer le regard et la vision de l'insoutenable horreur à l'aide de " verres de couleur », " des verres roses, rouges, bleus, des vitres magiques, des vitres de paradis ", des "vitres qui fassent voir la vie en beau ». II est probable que la guerre de 14 ait poussé à son paroxysme une certaine fusion orgiaque de l'archaïsme et de la modernité, qui s'inscrivait au cœur de l'esthétisation de la vie. II ne s'agit nullement de perdre la mémoire, mais, au-delà de ce qu'il est coutume d'appeler "le devoir de mémoire ", donner à cette guerre insensée le seul sens qu'elle puisse avoir : "C'est à ce niveau seulement que la guerre pouvait avoir un sens, au niveau de la souffrance individuelle ». Telle est l'affirmation portée en 1989 par Modris Eksteins, après qu'il s'est longuement intéressé à la genèse et au succès immédiat du livre d'Erich Maria Remarque, À l'Ouest rien de nouveau, paru en 1929. Dix ans après la fin des combats, ce livre pacifiste répondait aux attentes du public européen en manque de « textes vrais ». Modris Eksteins (1991: 335) conclut :

La guerre était affaire d'expérience personnelle, et non d'expérience collective. Elle s'apparentait désormais davantage à l'art qu'à l'Histoire.

Et c'est essentiellement par les discours proposés par l'exceptionnelle créativité artistique pluridisciplinaire du symbolisme et de tous les mouvements qui ont bourgeonné et fleuri en se nourrissant de sa sève que l'histoire humaine, celle de tout un chacun, se réaccordera à l'Histoire sociale. 


\section{Bibliographie sélective}

Apollinaire, Guillaume (1991), " L'Esprit et les poètes », CEuvres en prose complètes, t. II, Gallimard, Paris.

Baudelaire, Charles (1976), CEuvres complètes, Texte établi, présenté et annoté par Claude Pichois, Gallimard, Paris.

Bergez, Daniel (2008), Peindre, écrire, Le Dialogue des Arts, De la Martinière, Paris.

Denizeau, Gérard (2008), Le Dialogue des Arts [1874-2000], Larousse, Paris.

Eksteins, Modris (1989), Rites of Spring: The Great War and the Birth of the Modern Age, 1989, trad. Martine Leroy-Bastelli (1991), Le Sacre du Printemps : la Grande Guerre et la Naissance de l'Epoque moderne, Plon, Paris.
Éluard, Paul (1968), Capitale de la douleur, « Nouveaux poèmes », « Ne plus partager », Gallimard, t. 1, Paris.

Gaudard, François Charles / Suarez, Modesta (2003), Formes discursives du témoignage, Éd. Universitaires du Sud, Toulouse.

Peyré, Yves (2008), Peinture et poésie, le dialogue par le livre, Gallimard, Paris. 